\title{
Mulberry Fruits Extract Mitigate Vascular Dementia
}

\author{
${ }^{1,3}$ Pratchaya Kaewkaen, ${ }^{2,3}$ Jintanaporn Wattanathorn, \\ ${ }^{2,3}$ Terdthai Thong-Un, ${ }^{2,3}$ Supaporn Muchimapura, ${ }^{2,3}$ Panakaporn Wannanond, \\ ${ }^{2,3}$ Wipawee Thukhummee, ${ }^{4}$ Wiroje Kaewrueng, ${ }^{4}$ Sathaporn Wongareonwanakij, \\ ${ }^{4}$ Sompong Kraipoch, ${ }^{4}$ Prateep Meesilp
}

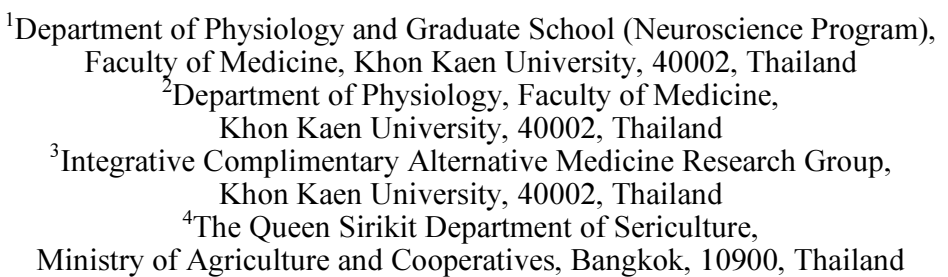

Received 2012-03-05, Revised 2012-08-09; Accepted 2012-08-31

\begin{abstract}
To date, Vascular Dementia (VaD) is the second most commonly found dementia following Alzheimer's disease. Though VaD is still increasing its importance, the therapeutic efficacy is still not in satisfaction level. Therefore, the searching for an acceptable cheap and safe neuroprotective agent is in a great demand. Based on the neuroprotective and cognitive enhancing effects in alcohol neurotoxicity of mulberry fruits, we hypothesized that mulberry fruits might attenuate memory impairment and brain damage in vascular dementia. Therefore, this study was set up to determine the effects and possible mechanism of actions of mulberry fruits. Male Wistar rats had been orally given mulberry fruits at doses of 2,10 and $50 \mathrm{mg} \cdot \mathrm{kg}^{-1} \mathrm{BW}$ for 7 days before and 21 days after the occlusion of right Middle Cerebral Artery (Rt. MCAO). Rats were evaluated spatial memory using Morris water maze every 7 days after Rt.MCAO throughout 21-day experimental period, then they were sacrificed for determined the density of cholinergic neuron in hippocampus and determine acetyl cholinesterase (AChE) enzyme, a key enzyme indicating Acetylcholine (ACh) turnover in hippocampus and assessment of brain infarction volume The results show that the mulberry fruits extract significantly improved memory performance in Morris water maze test, number of cholinergic neurons and decrease the level of acetyl cholinesterase activity. The mulberry fruit extract is the potential neuroprotective agent and cognitive enhancing fruits. However, further researches are required identify the possible active ingredient and precise underlying mechanism.
\end{abstract}

Keywords: Mulberry Fruit, Vascular Dementia, Stroke

\section{INTRODUCTION}

Vascular dementia (VaD) is the in elderly population (Malouf and Birks, 2004). It is caused by chronic reduced blood flow to the brain. The most common cause appears to be stroke or series of strokes. It has been reported that $79.5 \%$ of vascular dementia patient had a history of stroke (Lindsay et al., 1997). When the blood supply carrying oxygen and nutrients to the brain is interrupted by a blocked or diseased vascular system, the neurodegeneration occur in the affected brain area and the gives rise to a progressive decline in memory and cognitive functioning. It was found that level of oxidative stress markers such as Malondialdehyde (MDA) and 8-hydroxydeoxyguanosine (8-OHdG) (Shi et al., 2012) were elevated while the antioxidant systems such as the level of vitamin E (Ryglewicz et al., 2002) were decreased in $\mathrm{VaD}$ patients (Gustaw-Rothenberg et al., 2010). In addition, the cholinergic system was also reported to involve in cognitive impairment in $\mathrm{VaD}$ (Roman, 2005; Wang et al., 2009).

Current approaches to dementia-related neurodegenerative diseases still highly rely on relieving symptoms. Moreover, medications against this condition are very expensive. Therefore, there is a great demand of

Corresponding Author: Jintanaporn Wattanathorn, Department of Physiology, Faculty of Medicine, Khon Kaen University, 40002, Thailand Tel: 66-43-348394 
an acceptable cheap and safe neuroprotective agent. Based on the crucial role of oxidative stress and cholinergic system on $\mathrm{VaD}$ mentioned earlier, the beneficial effect of substance possessing antioxidant and acetylcholinesterase inhibitory effects have been considered.

Morus alba or mulberry belongs to the Moraceae family. It has been widely planted in both the Northeast and North of Thailand. Mulberry fruit is widely regarded as a nutritious food. It can be eaten freshly or widely used in the production of wine, fruit juice, jam and canned food. Mulberry fruit has been long term used as medicine. According to Chinese Materia Medica, mulberry fruit has been used as blood tonic to nourish the vin and blood and as anti-aging ( $\mathrm{Li}$ and Luo, 2003). It is also used for treating weakness, fatigue, anemia and premature graying of hair. Recently, several lines of evidence have demonstrated that mulberry fruits can protect against brain damage in various conditions including Parkinson's disease (Kim et al., 2010), cerebral ischemia (Kang et al., 2006) and alcohol neurotoxicity. In addition, the memory impairment and the elevated acetyl cholinesterase induced by alcohol toxicity are also reversed. Based on the cognitive enhancing effect and neurotective effect against alcohol neurotoxicity of mulberry fruits mentioned earlier, we hypothesized that mulberry fruits might provide beneficial effects to attenuate memory impairment and brain damage in vascular dementia. Therefore, this study was set up to determine the effects and possible mechanism of actions of mulberry fruits.

\section{MATERIALS AND METHODS}

\subsection{Plant Material and Extraction \\ 2.1.1. Animals}

Adult male Wistar rats $(300-350 \mathrm{~g}, 8$ weeks old) were obtained from National Laboratory Animal Center, Salaya, Nakorn Pathom and were housed in group of 5 per cage in standard metal cages at $22 \pm 2^{\circ} \mathrm{C}$ on $12: 12 \mathrm{~h}$ light-dark cycle. All animals were given access to food and water ad libitum. The experiments were performed to minimize animal suffering in accordance with the internationally accepted principles for laboratory use and care of European Community (EEC directive of 1986; 86/609/EEC). The experimental protocols were approved by the Institutional Animal Care and Use Committee (AEKKU 1/2552).

\subsection{Drugs}

Mulberry fruits were extract from The Queen Sirikit Department of Seri culture, Ministry of Agriculture and Cooperative, Thailand. Both Donepezil (Aricept ${ }^{\mathbb{B}}$ ), a standard drug used for mild cognitive impairment treatment and vitamin $\mathrm{C}$, an antioxidant possessing the neuroprotective and cognitive enhancing effects were used as positive controls in this study. Propylene Glycol (PG) was used as vehicle throughout the study. All administered substances were freshly prepared.

\subsection{Plant Preparation}

All mulberry fruits used in this study is prepared and provided by The Queen Sirikit Department of Seri Culture, Thailand. Mulberry fruits were collected from the Queen Sirikit Seri Culture Center Udon Thani. All berries were picked at the commercially ripen stage and selected according to uniformity color. Then, the fruits were dried at $70^{\circ} \mathrm{C}$ for 4 days and grounded to powder. Then, 4 kilograms of mulberry fruit powder were extracted 3 times with ethyl alcohol 5 liters per time by percolation techniques. The obtained extracts were evaporated under reduced pressure to yield $7.37 \%$ of ethanol extract.

\subsection{Experimental Protocol}

All rats were randomly divided into 6 groups. Each group contained 6 rats.

\section{Group 1}

Vehicle treated group. The animals in this group were treated with Propylene Glycol (PG)

\section{Group 2-3}

Positive control treated group. The positive control group was treated with the standard drugs used for treating the related the condition. Vitamin C, a wellknown antioxidant, was orally administered at dose of $250 \mathrm{~kg}^{-1} \mathrm{BW}$. In the determination of cognitive function, the positive control group was treated with Donepezil (Aricept ${ }^{\circledR}$, a cholinesterase inhibitor) at dose of $1 \mathrm{mg} \mathrm{kg}^{-1} \mathrm{BW}$.

\section{Group 4-6}

Mulberry fruits extract treated group. The animals in group 4-6 were treated with the Mulberry fruits extract at various doses ranging from 2,10 and $50 \mathrm{mg} \mathrm{kg}^{-1}$ BW respectively via oral route administration for a week once daily throughout the experimental period and three weeks after induce middle cerebral artery occlusion.

All animals were treated with vehicle, positive control or mulberry fruits extract at a period of 7 days before and 21 days after right Middle Cerebral Artery Occlusion (MCAO).

\subsection{Surgical Procedure to Induction of Middle Cerebral Artery Occlusion (MCAO)}

Focal cerebral ischemia was performed according to modified method of (Longa et al., 1989). In brief, rats were anesthetized by thiopental sodium at dose of $50 \mathrm{mg} \mathrm{kg}^{-1}$ $\mathrm{BW}$. The right common carotid artery and the right external carotid artery were exposed through a ventral midline neck incision and were ligated proximally. A silicone coated nylon monofilament (4-0) suture (USS DGTM sutures; Tyco Healthcare group LP, Connecticut, USA) with its tip 
rounded by heating near a flame was inserted through an arteriectomy in the common carotid artery just below the carotid bifurcation and then advanced into the internal carotid artery approximately $17-18 \mathrm{~mm}$ distal to the carotid bifurcation until a mild resistance was felt. Occlusion of the origins of the anterior cerebral artery, the middle cerebral artery and the posterior communicating artery was thereby achieved. Then, the wound was sutured, the rats were returned to their cages with free access to food and water. The incision sites were infiltrated with $10 \%$ PovidoneIodine Solution for anti-septic postoperative care.

\subsection{Assessment of Cognitive Function}

Animals were tested spatial memory by the water maze test (Morris et al., 1982). The apparatus was a pool with 170 $\mathrm{cm}$ diameter filled up with tap water for $40 \mathrm{~cm}$ deep and the water surface was covered with nontoxic powder. The pool was divided into four quadrants and the removable escape platform was placed in the center on one quadrant below the water level. For animals, the location of the platform was invisible and it remained there throughout the training. The animals must memorize the environment cues to locate the platform. Each animal was placed in the water in the starting quadrant and allowed to swim until it found and climbed onto the platform. The time for animal to reach the hidden platform was recorded as escape latency or acquisition time.

\subsection{Acetylcholinesterase Assay}

After the animals were sacrificed, brains were isolated and kept cool in ice buckets. Then the tissues of hippocampus were homogenized in 4 volume of $1.15 \% \mathrm{KCl}$ with glass Potter-Elvehjim homogenizer (Trounce, 1973). An AChE assay was performed using the colorimetric method (Ellman et al., 1961) with minor modifications. The hippocampus was homogenized in $0.1 \mathrm{M}$ phosphate buffer, $\mathrm{pH}$ 8. The reaction mixture consist of $2.6 \mathrm{~mL}$ of phosphate buffer (0.1M, pH 8.0)0.4 ml aliquot of homogenate and 0.1 $\mathrm{mL}$ of $0.01 \mathrm{M}$ dithiobisnitrobenzoic Acid (DTNB). After the addition of the substrate acetylthiocholine iodine $(0.075$ $\mathrm{M})$ change in the absorbance was noted every 2 min for 10 min at $412 \mathrm{~nm}$ using a spectrophotometer. The activity was expressed as micromoles hydrolyzed per milligram protein of tissue $\left(\mu \mathrm{mg}^{-1}\right.$.protein)

\subsection{Immunohistochemical Staining Cholineacetyltransferase (ChAT)}

A series of sections containing hippocampus were reacted in a mouse monoclonal antibody directed against choline acetyltransferase (ChAT) (Chemicon Internation, Inc., CA, USA) and a modification of a previously described protocol employing the DAKO Strept ABC Complex/HRP duet kit. In brief, the sections were eliminated endogenous peroxidase activity by $0.5 \% \mathrm{H}_{2} \mathrm{O}_{2}$ in methanol. Sections were washed in running tap water and distilled water for $1 \mathrm{~min}$ each, then rinsed in KPBS and
KPBS-BT for 5 min per each process. Excess buffer was removed and then incubated for $30 \mathrm{~min}$ in a blocking solution composed of $5 \%$ normal goat serum in KPBS-BT. Then, the sections were incubated in mouse primary antibody against ChAT diluted 1: 100 in KPBS-BT at room temperature for $2 \mathrm{~h}$ and incubate at $4^{\circ} \mathrm{C}$ for $48 \mathrm{~h}$. The tissue was rinsed in KPBS-BT ( 2 washes $\times 7 \mathrm{~min}$ ), incubated for 1 $\mathrm{h}$ in biotinylated goat antimouse $\mathrm{IgG}$ antibody, rinsed in KPBS-BT ( 2 washes x 7 min) and then incubated in Strept $\mathrm{ABC}$ Complex/HRP for $4 \mathrm{~h}$. The sections were rinsed in KPBS-BT (1 min) and KPBS (2 washes x $10 \mathrm{~min}$ ). ChAT immunoreactivity was visualized using $0.025 \%$ 3, 3' diaminobenzedine (DAB, Sigma) and $0.01 \% \mathrm{H}_{2} \mathrm{O}_{2}$. For 24 $\mathrm{h}$. Finally, sections were rinsed in running tap water, air dried and cover-slipped using permount.

\subsection{Morphological Analysis}

Five coronal sections of each rat in each group were studied quantitatively. Neuronal counts in hippocampus were performed by eye using a 40x magnification with final field $255 \mu^{2}$ according to the following stereotaxic coordinates: AP $-4.8 \mathrm{~mm}$, lateral $\pm 2.4-6 \mathrm{~mm}$, depth 3-8 mm. The observer was blind to the treatment at the time of analysis. Viable stained neurons were identified on the basis of a stained soma with at least two visible processes. Counts were made in five adjacent fields and the mean number extrapolated to give total number of neurons per $255 \mu \mathrm{m}^{2}$. All data are represented as number of neurons per $255 \mu \mathrm{m}^{2}$.

\subsection{Statistical Analysis}

Data were presented as mean \pm Standard Error of Mean (SEM). The analysis was performed using oneway Analysis of Variance (ANOVA), followed by LSD test. All statistical results were considered significant at $\mathrm{p}$-value $<0.05$.

\section{RESULTS}

\subsection{Effect of mulberry Fruits Extract in Animal Model of Stroke in Morris Water Maze Test}

Cognitive deficit is commonly clinical sign in vascular dementia. In this study examined the effect of mulberry fruit extract on spatial memory both healthy condition and stroke condition, after induce middle cerebral artery occlusion. Data were recorded escape latency and retention time. In healthy condition, the results showed significant changes in escape latency and retention times in rats which received either vitamin $C$ or Aricept ${ }^{\circledR}$. Moreover, mulberry fruit significantly decreased escape latency time in dose $50 \mathrm{mg} \mathrm{kg}^{-1} \mathrm{BW}$ in single dose administration and increased retention time in mulberry fruit at dose of $2,10 \mathrm{mg} \mathrm{kg}^{-1} \mathrm{BW}$ on single dose ( $p$-value $<0.05$, all; compared with vehicle treated groups) (Fig. 1-2). 


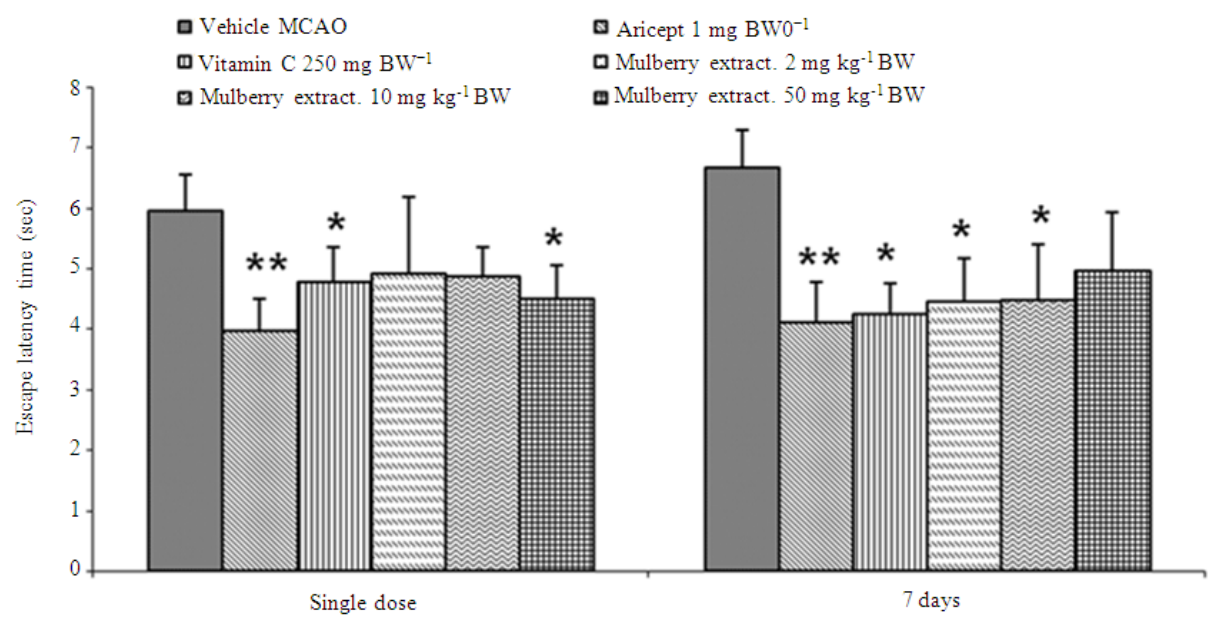

Fig. 1. The effect of mulberry fruit extract on escape latency time in healthy condition. Rats were treated with vehicle, Aricept $\AA$, vitamin $C$ or the mulberry fruit extract at various doses ranging from 2,10 and $50 \mathrm{mg} \mathrm{kg}^{-1}-1 \mathrm{BW}$ via oral route for 7 days, then

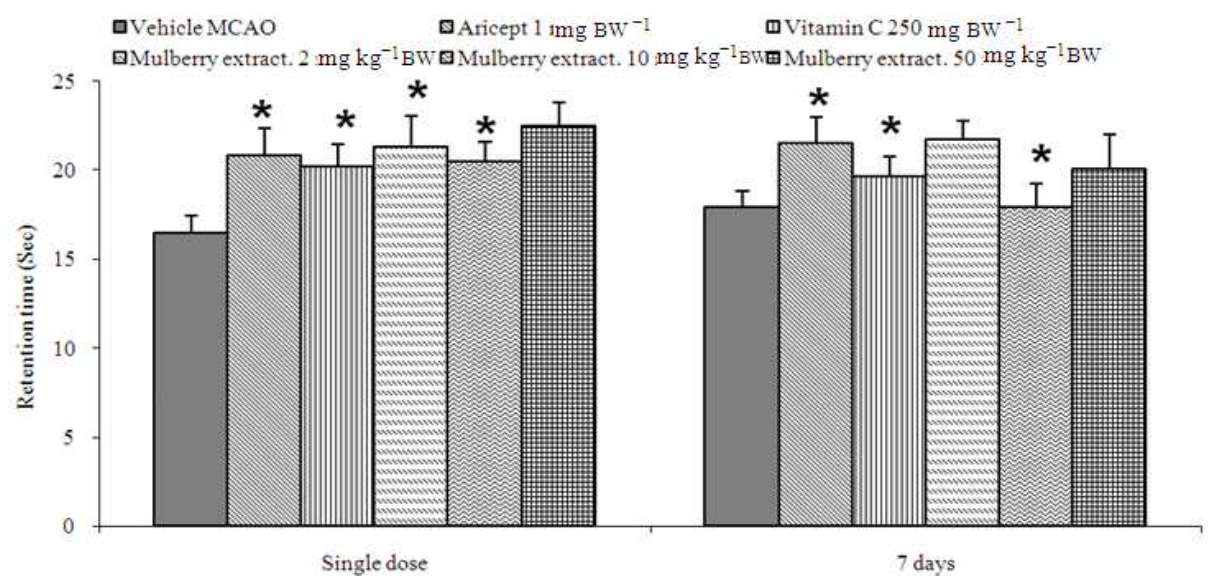

Fig. 2. The effect of mulberry fruit extract on retention time in healthy condition. Rats were treated with vehicle, Aricept $\mathbb{R}$, vitamin $\mathrm{C}$ or the mulberry fruit extract at various doses ranging from 2,10 and $50 \mathrm{mg} \mathrm{kg}^{-1}-1 \mathrm{BW}$ via oral route for 7 days, then they

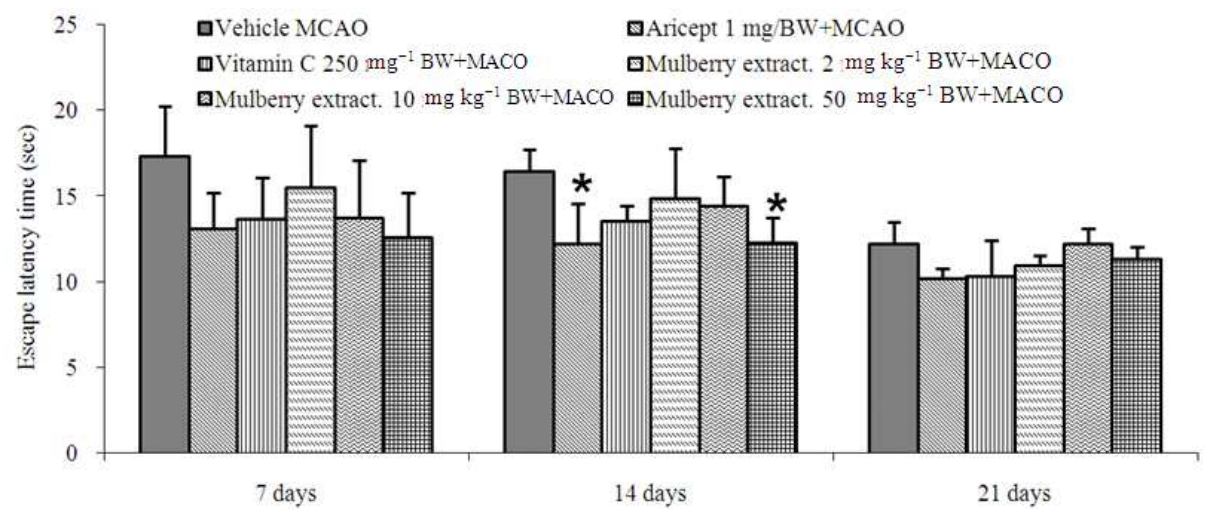

Fig. 3. The effect of mulberry fruit extract on escape latency time in stroke condition. Rats were treated with vehicle, Aricept ${ }^{\circledR}$, vitamin $C$ or the mulberry fruit extract at various doses ranging from 2,10 and $50 \mathrm{mg} \mathrm{kg}^{-1}-1 \mathrm{BW}$ via oral route for 21 days, then 


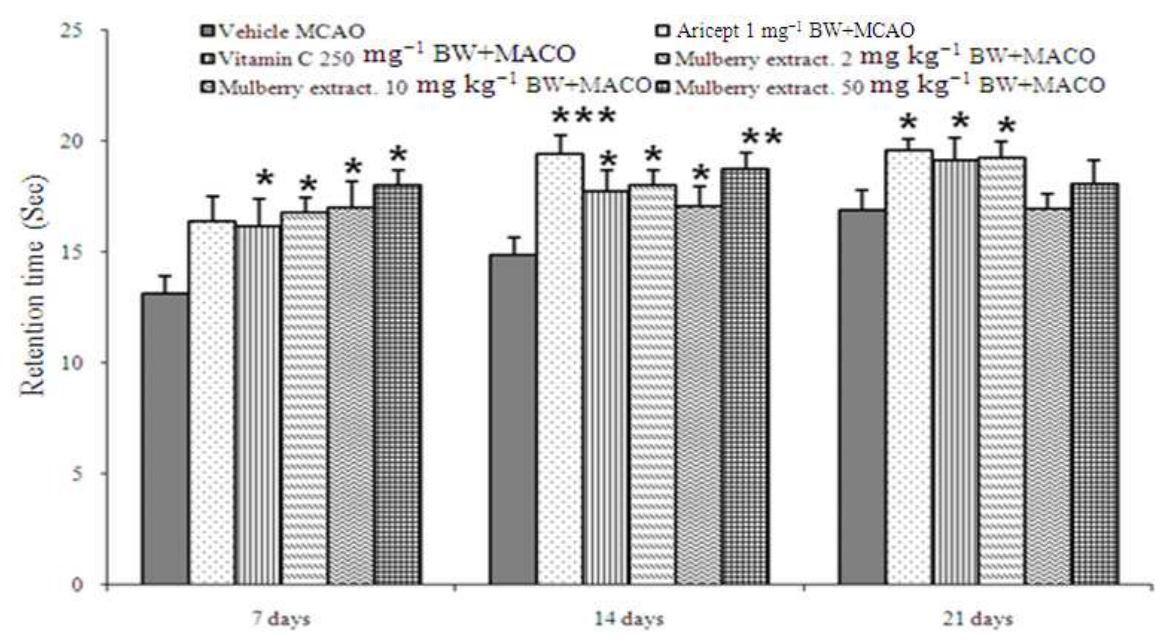

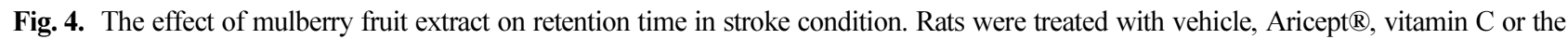
mulberry fruit extract at various doses ranging from 2,10 and $50 \mathrm{mg} \mathrm{kg}^{-1}-1 \mathrm{BW}$ via oral route for 21 days, then they

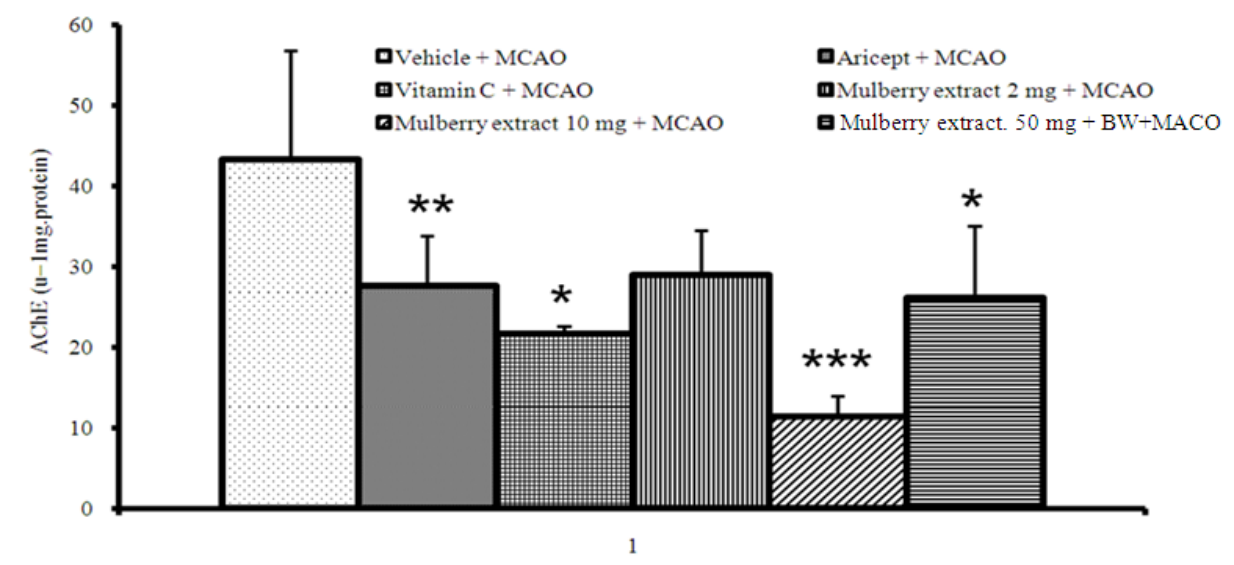

Fig. 5. The effect of mulberry fruit extract on the alteration of acetylcholinesterase enzyme activity in hippocampus. Rats were treated with vehicle, Aricept ${ }^{\circledR}$, vitamin $\mathrm{C}$ and various doses of mulberry fruit extract via oral route for 7 days, then they $\mathrm{w}$

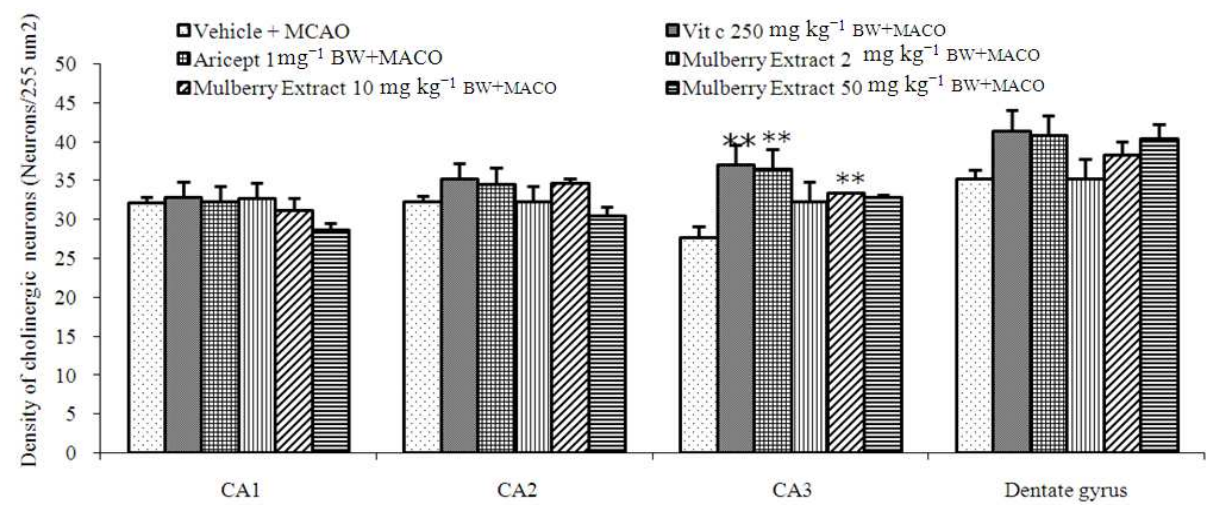

Fig. 6. The effect of mulberry fruit extract on alteration of density of cholinergic neuron in various subregion of hippocampus. Data are presented as mean $\pm \operatorname{SEM}$ ( $n=6 /$ group $) *$ p-value $<0.05$ compared with vehicle plus MCAO group 
Table 1. Effect of Aricept ${ }^{\circledR}$, vitamin $\mathrm{C}$ and various doses of mulberry fruit extract on motor function using neurological score. Values given are the mean $\pm \operatorname{SEM}(n=6)$

\begin{tabular}{|c|c|c|c|c|}
\hline \multirow[b]{2}{*}{ Group/day } & \multirow[b]{2}{*}{ Pre surgery } & \multicolumn{3}{|l|}{ Post surgery } \\
\hline & & 7 days & 14 days & 21 days \\
\hline Vehicle + MCAO & $5.00 \pm 0.00$ & $2.87 \pm 0.23$ & $3.50 \pm 0.20$ & $3.75 \pm 0.21$ \\
\hline Aricept ${ }^{\circledR}+$ MCAO & $5.00 \pm 0.00$ & $3.5 \pm 0.22$ & $4.25 \pm 0.18 * *$ & $4.75 \pm 0.15 * * *$ \\
\hline Vitamin $\mathrm{C}+\mathrm{MCAO}$ & $5.00 \pm 0.00$ & $4.5 \pm 0.92 * * *$ & $4.62 \pm 0.34 * * *$ & $4.87 \pm 0.27 * * *$ \\
\hline Mulberry extract $2 \mathrm{mg} \mathrm{kg}^{-1} \mathrm{BW}+\mathrm{MCAO}$ & $5.00 \pm 0.00$ & $3.87 \pm 0.21 *$ & $4.25 \pm 0.17 * * *$ & $4.75 \pm 0.15 * * *$ \\
\hline Mulberry extract $10 \mathrm{mg} \mathrm{kg}^{-1} \mathrm{BW}+\mathrm{MCAO}$ & $5.00 \pm 0.00$ & $4.12 \pm 0.20 * * *$ & $4.62 \pm 0.17 * * *$ & $4.87 \pm 0.14 * * *$ \\
\hline Mulberry extract $50 \mathrm{mg} \mathrm{kg}^{-1} \mathrm{BW}+\mathrm{MCAO}$ & $5.00 \pm 0.00$ & $3.00 \pm 0.23$ & $4.62 \pm 0.15 * * *$ & $4.75 \pm 0.15 * * *$ \\
\hline
\end{tabular}

$*, * *, * * * \mathrm{p}$-value $<0.05 ; .01$ and .001 respectively ; compared with vehicle plus MCAO

In stroke condition, Vitamin C, Aricept ${ }^{\circledR}$ and mulberry fruit extract extract at dose $50 \mathrm{mg} \mathrm{kg}{ }^{-1} \mathrm{BW}$ significantly decreased the escape latency time in Morris water maze test after MCAO 7 days after MCAO when compared to the vehicle plus MCAO group. Moreover, the significant effect of mulberry fruit extract at dose $2,10,50 \mathrm{mg} \mathrm{kg}^{-1} \mathrm{BW}$ was observed in retention time of Morris water maze test after 7 and 14 days of post MCAO but no significant was observed in 21 days post MCAO (Fig. 3-4). Vitamin C treated rats significantly increased neurological score throughout the experimental period ( $\mathrm{p}$-value $<.001$ all; compared with vehicle plus MCAO; compared with vehicle plus MCAO). All doses of mulberry fruit extract significantly improved neurological score throughout the experimental period except the high dose of mulberry fruit extract which started to show the significant improvement at 14 days of treatment until the end of experimental period. (p-value $<0.001$ all; compared with vehicle plus MCAO) (Table 1).

\subsection{Effect of Mulberry Fruit Extract on Acetylcholineasterse Activity}

The cholinergic system plays the crucial role in spatial memory, the effect of mulberry fruit extract on the activity of AChE, an indirect indicator of cholinergic function was evaluated. It was found that the mulberry fruit extract at 10 and $50 \mathrm{mg}^{-1} \mathrm{~kg} \mathrm{BW}$, vitamin $\mathrm{C}$ and Aricept ${ }^{\circledR}$ significantly decreased the AChE activity in the hippocampus homogenate ( $\mathrm{p}$-value $<0.05$ compared to vehicle $+\mathrm{MCAO}$ ) (Fig. 3). However, the low doses $\left(2 \mathrm{mg} \mathrm{kg}{ }^{-1} \mathrm{BW}\right.$ respectively) of mulberry fruit extract failed to show significant alterations on this enzyme activity (Fig. 5).

\subsection{Effect of Mulberry Fruit Extract on Cholinergic Neuron Density}

The alteration of density of Cholineacetyl Transferees (ChAT) positive stained neurons in various sub-regions of hippocampus after induction of MCAO, The medium dose of mulberry fruit extract $\left(10 \mathrm{mg} \mathrm{kg}^{-1} \mathrm{BW}\right)$ significantly enhanced cholinergic neurons density in CA3 $(\mathrm{P}<0.01$, compared to vehicle plus MCAO) (Fig. 6).

\section{DISCUSSION}

In this study, we have demonstrated the neuroprotective effect of Mulberry fruits extract via oral route administration in the vascular dementia model rats. Our data showed that mulberry fruits decreased the escape latency time and increased retention time of Morris water maze test. The neurological score was increased. The number of cholinergic neurons was increases in hippocampus CA3 and decreased the level of acetyl cholinesterase activity when compare with vehicle treated groups.

The cognitive enhancing effect observed in this study associated with the inhibition of acetylcholinesterase in hippocampus, an area which played a crucial role in learning and memory. Acetyl cholinesterase inhibitors use as drug in mild cognitive impairment and behavioral symptoms of Alzheimer's disease with cerebrovascular disease and vascular dementia. For Donepezil (Aricept ${ }^{\mathbb{B}}$ ) is a drug that moderate the acetylcholine response by inhibiting acetylcholinesterase enzyme. It has been suggested that could produce a beneficial cascade of neurotransmitters in the brains of dementia patients (Maelicke, 2000).

Mulberries (Morus alba Linn.) are anthocyanins rich fruits, which are a traditional Chinese medicine. The major anthocyanins identified in the fruit extract are cyanidin 3glucoside and cyanidin 3-rutinoside (Suh et al., 2003). Recently, mulberry fruit has been reported to possess medicinal benefit, such as potent anti-oxidant activity (Chen et al., 2005; Kang et al., 2006), anti-inflammation (Shin et al., 2006), anti-thrombotic (Allen and Bayraktutan, 2009). Moreover, mulberry exhibit inhibitory effect on the migration and invasion of a human lung cancer cell line (Chen et al., 2005). Therefore, exploring the diverse bioactivities of this extract can be exploited pharmacologically resulting to candidate for decreasing the aliments associated with stroke. It had been shown that the memory impairment was involved with the neuronal damage in brain (Mori et al., 1997). Therefore, the neuron density and cholinergic neurons density were also investigated in hippocampus. Mulberry fruit extract could significantly increase neurons density in CA3 hippocampus while no significant changes in CA1, CA2 and dentate gyrus. 
Based on this finding and the crucial role of cholinergic system on learning and memory, the cognitive enhancing effect particularly the spatial memory of mulberry fruit extract might be related to the increase cholinergic function in hippocampus. So, the exploring of this extract can be exploited pharmacologically resulting to candidate for decreasing the aliments associated with stroke.

\section{CONCLUSION}

In summary, we conclude that, this study provides experimental evidence for the potential effect of mulberry fruits extract to enhance cognitive effect in model of vascular dementia. But further studies are still required.

\section{ACKNOWLEDGEMENT}

This study was supported by the Integrative Complementary Alternative Medicine Research Group, Faculty of Medicine, Thailand and The Queen Sirikitti Department of Sericulture, Ministry of Agriculture and cooperative, Bangkok, Thailand.

\section{REFERENCES}

Allen, C.L. and U. Bayraktutan, 2009. Oxidative stress and its role in the pathogenesis of ischaemic stroke. Int. J. Stroke, 4: 461-70. DOI: 10.1111/j.17474949.2009.00387.x

Chen, C.C., L.K. Liu, J.D. Hsu, H.P. Huang and Yang, 2005. Mulberry extract inhibits the development of atherosclerosis in cholesterol-fed rabbits. Food Chem., 91: 601-607. DOI: 10.1016/j.fooodchem.2004.06.039

Ellman, G.L., K.D. Courtney, V. Andres and R.M. Featherstone, 1961. A new and rapid colorimetric determination of acetylcholinesterase activity. Biochem. Pharmacol., 7: 88-95. PMID: 13726518

Gustaw-Rothenberg, K., K. Kowalczuk and M. Stryjecka-Zimmer, 2010. Lipids' peroxidation markers in Alzheimer's disease and vascular dementia. Geriatr. Gerontol. Int., 10: 161-166. DOI: 10.1111/j.1447-0594.2009.00571.x

Kang, T.H., J.Y. Hur, H.B. Kim, J.H. Ryu and S.Y. Kim, 2006. Neuroprotective effects of the cyanidin-3-O$\beta$-d-glucopyranoside isolated from mulberry fruit against cerebral ischemia. Neurosci. Lett., 391: 122126. DOI: 10.1016/j.neulet.2005.08.053

Kim, H.G., M.S. Ju, J.S. Shim, M.C. Kim and S.H. Lee et al., 2010. Mulberry fruit protects dopaminergic neurons in toxin-induced Parkinson's disease models. Br. J. Nutr., 104: 8-16. DOI: 10.1017/S0007114510000218
Li, S. and X. Luo, 2003. Compendium of Materia Medica. 1st Edn., Foreign Languages Press, Beijing, ISBN-10: 7119032607, pp: 4397.

Lindsay, J., R. Hebert and K. Rockwood, 1997. The Canadian study of health and aging: Risk factors for vascular dementia. Stroke, 28: 526-553. DOI: 10.1161/01.STR.28.3.526

Longa, E.Z., P.R. Weinstein, S. Carlson and R. Cummins, 1989. Reversible middle cerebral artery occlusion without craniectomy in rats. Stroke, 20: 84-91. DOI: 10.1161/01.STR.20.1.84

Maelicke, A. 2000. Allosteric modulation of nicotinic acetylcholine receptors as a treatment strategy for Alzheimer's disease. Dement Geriatr. Cogn. Disord., 11: 11-18. DOI: 10.1016/S00142999(00)00093-5

Malouf, R. and J. Birks, 2004. Donepezil for vascular cognitive impairment. Cochrane Database Syst. Rev. PMID: 14974068

Mori, E., Y. Yoneda, H. Yamashita, N. Hirono and M. Ikeda et al., 1997. Medial temporal structures relate to memory impairment in Alzheimer's disease: An MRI volumetric study. J. Neurol. Neurosurg. Psychiatry, 63: 214-221. PMID: 9285461

Morris, R.G., P. Garrud, J.N. Rawlins and J. O'Keefe, 1982. Place navigation impaired in rats with hippocampal lesions. Nature, 297: 681-683. PMID: 7088155

Ryglewicz, D., M. Rodo, P.K. Kunicki, M. BednarskaMakaruk and A. Graban et al., 2002 Plasma antioxidant activity and vascular dementia. J Neurol. Sci., 203: 195-197. DOI: 10.1016/S0022510X(02)00290-3

Roman, G.C., 2005. Cholinergic dysfunction in vascular dementia. Curr. Psychiatry Rep., 7: 18-26. DOI: $10.1007 / \mathrm{s} 11920-005-0019-2$

Shi, G.X., C.Z. Liu, L.P. Wang, L.P. Guan and S.Q. Li, 2012. Biomarkers of oxidative stress in vascular dementia patients. Can. J. Neurol. Sci., 39: 65-68. PMID: 22384487

Shin, W.H., S.J. Park and E.J. Kim, 2006. Protective effect of anthocyanins in middle cerebral artery occlusion and reperfusion model of cerebral ischemia in rats. Life Sci., 79: 130-137. PMID: 16442129

Suh, H.J., D.O. Noh, C.S. Kang, J.M. Kim and S.W. Lee, 2003. Thermal kinetics of color degradation of mulberry fruit extract. Food Nahrung., 47: 132-135. DOI: 10.1002/food.200390024

Trounce, J.R., 1973. Fundamentals of drug metabolism and drug disposition. Proc. R Soc. Med., 66: 78-78.

Wang, J., H.Y. Zhang and X.C. Tang, 2009. Cholinergic deficiency involved in vascular dementia: Possible mechanism and strategy of treatment. Acta Pharmacol. Sin., 30: 879-888. PMID: 19574993 